\title{
Infección crónica por Pseudomonas aeruginosa en pacientes con bronquiectasias no fibrosis quística
}

\author{
PAULINA TRUJILLO M.*, PATRICIA FERNÁNDEZ V.***, FRANCISCO ARANCIBIA H.*, \\ BAIRD ZEGPI KELLER*, GABRIEL CAVADA CH.**, VICENTE LOAYZA F.**, \\ PEDRO PALZA C.**, MARÍA VERGARA DE C.** y PEDRO CARRASCO G.*
}

\section{Chronic infection by Pseudomonas aeruginosa in patients with non-cystic fibrosis bronchiectasias}

Introduction: Chronic airways infection with Pseudomonas aeruginosa (PA) is a common situation in patients with Bronchiectasis $(B Q)$ and constitutes a breakdown in the natural history of the latter. Moreover, BQ is also associated with a poor prognosis and an increased severity of the disease. Objective: To describe the characteristics of the population diagnosed with non-Cystic Fibrosis Bronchiectasis (non-CFB) who are chronically infected with $P A$, and to perform a comparison with patients with negative sputum cultures. Methodology: We performed a retrospective analysis of the clinical files of patients diagnosed with non-CFB who were attended at the 'Instituto Nacional del Tórax' (Chile) between July 2007 and April 2017. The characteristics of the population were described and the FACED scores and other severity indexes were compared. Results: The average age of patients was $55 \pm$ 17.3 years-old, and $81 \%$ of them were female. According to PA isolation in sputum culture, they were classified as "chronically infected" (non-CFB with PA, $n=61$ ) and "not infected with PA" (non-CFB without $P A, n=59)$. There were no differences in age and gender between the two groups. On the other hand, FEV $V_{1}$ was lower in the non-CFB PA group. The calculated FACED score was higher in colonized patients. The most frequent etiology was post-infectious, mainly TB sequels, with a $30.8 \%$ unidentified etiology. Conclusions: Patients with bronchiectasis chronically infected with Pseudomonas aeruginosa show increases in the severity of the disease, with a lower FEV $V_{1}$ and a higher FACED score. The postinfectious etiology is highlighted in our group.

Key words: Pseudomonas aeruginosa; bronchiectasis; Pseudomonas infections; respiratory function tests.

\section{Resumen}

Introducción: La infección crónica por Pseudomonas aeruginosa (PA) es frecuente en pacientes con bronquiectasias $(B Q)$ y representa un quiebre en la historia natural de la enfermedad, asociándose a mal pronóstico y mayor severidad. Objetivo: Caracterizar la población portadora de $B Q$ no fibrosis quística (BQ no-FQ) del Instituto Nacional del Tórax (INT) infectados crónicamente con PA comparándolos con pacientes que mantienen cultivos de expectoración negativos para este germen. Metodología: Revisión retrospectiva de fichas clínicas de pacientes portadores de BQ del INT entre julio de 2007 y abril de 2017. Se caracterizó la población y se comparó score de FACED y otros índices de gravedad. Resultados: El promedio de edad fue de $55 \pm 17,3$ años, $81 \%$ de los pacientes fue de género femenino. De acuerdo a aislamiento de Pseudomonas en cultivo esputo se clasificaron como infectados crónicamente (BQ con $P A ; n=61)$ y no infectados con $P A(B Q \sin P A ; n=59)$. No hubo diferencias entre los grupos en edad y sexo. El VEF fue más bajo en el grupo con PA los que tienen más hospitalizaciones. Se calculó el índice de riesgo FACED siendo mayor en los pacientes colonizados. La etiología más frecuente es la postinfecciosa, principalmente secuelas de TBC, con 30,8\% de etiología no identificada. Conclusiones: Los pacientes con bronquiectasias con infección crónica por Pseudomonas aeruginosa tienen una enfermedad más severa, con VEF ${ }_{1}$ más bajo, y con mayor índice de severidad de FACED. Destaca en nuestro grupo la etiología postinfecciosa.

Palabras clave: Pseudomonas aeruginosa; bronquiectasias; infección por Pseudomonas; pruebas de función respiratoria.

\footnotetext{
* Instituto Nacional del Tórax.

** Universidad Finis Terrae.
} 


\section{Introducción}

Las bronquiectasias no-fibrosis quística (BQ no-FQ) corresponden a una enfermedad respiratoria crónica caracterizada por un síndrome clínico de tos, producción de esputo e infección bronquial recurrente que se evidencian radiológicamente por la dilatación anormal y permanente de los bronquios ${ }^{1}$. En esta definición se excluyen a los pacientes con fibrosis quística pues tienen una fisiopatología diferente y manejo específico.

Se reconoce como una enfermedad frecuente, con una prevalencia variable según la población estudiada, que varía de 53 a 566 casos por 100.000 habitantes con predominio en el género femenino ${ }^{2}$.

Corresponden a un grupo heterogéneo, con diversas etiologías y distintos grados de severidad, que han motivado la investigación clínica y la creación de guías de manejo durante los últimos años, principalmente en Europa ${ }^{1}$, con importantes iniciativas latinoamericanas ${ }^{5-7}$, pero aún con muchas interrogantes por resolver, con un creciente desarrollo de la investigación de esta patología.

Es de gran interés identificar a aquellos pacientes que cursan con enfermedad más grave, siendo validado para la población latinoamericana el score de $\mathrm{FACED}^{7.8}$, que evalúa la edad, aspectos clínicos (disnea), función pulmonar $\left(\mathrm{VEF}_{1}\right)$, microbiología (infección crónica por Pseudomonas aeruginosa) y hallazgos radiológicos (número de lóbulos afectados en la tomografía computada), siendo un predictor de mortalidad a los 5 años desde el diagnóstico ${ }^{8}$.

Sin embargo, la infección crónica por Pseudomonas aeruginosa (PA) por sí sola es relevante. Si bien, se asocia a mal pronóstico ${ }^{9}$, se trata de una condición potencialmente tratable y que podría modificar la evolución de la enfermedad.

El objetivo de nuestro estudio es caracterizar la población adulta portadora de BQ no-FQ del Instituto Nacional del Tórax (INT) infectados crónicamente con PA comparándolos con pacientes que mantienen cultivos de expectoración negativos para este microorganismo.

\section{Metodología}

El Instituto Nacional del Tórax es un centro de referencia nacional de las patologías torácicas de alta complejidad. Cuenta con un registro único de ficha, un laboratorio de función pulmonar, laboratorio de microbiología y Servicio de Radiología, desde donde se obtuvieron los datos para este estudio. Se realizó un estudio retrospectivo observacional en el cual fueron incluidos 120 pacientes mayores de 15 años con diagnóstico de BQ no-FQ del INT entre julio de 2007 y abril de 2017. De la ficha clínica se obtuvo el diagnóstico de BQ no-FQ registrado por un especialista neumólogo del INT y confirmado por tomografía computada de tórax, además de la etiología, el $\mathrm{VEF}_{1}$ y el número de ingresos hospitalarios desde el primer cultivo de expectoración que resulta positivo para PA o desde el momento del diagnóstico para los casos en que no presentaron cultivos positivos para este germen. La presencia de PA en cultivos de expectoración de estos pacientes se corroboró con el laboratorio de microbiología y se catalogaron a los pacientes como "infectados crónicamente" si es que presentaban al menos dos cultivos de expectoración positivos para PA. Además, se revisaron las tomografías computadas de tórax para conocer el número de lóbulos comprometidos. Con estos datos, se calculó el score de FACED para cada paciente.

\section{Análisis estadístico}

Los resultados son expresados como: valores promedio \pm desviación estándar para variables en escala numérica y en porcentajes para medidas registradas en escala nominal. Las variables continuas fueron comparadas en pacientes con BQno FQ que tenían infección crónica por PA versus no infectados por PA, utilizando una prueba $t$ de student. Para las variables categóricas se utilizó la prueba de $\chi^{2}$ o prueba de Fisher. Para todas las pruebas, el nivel de significación se estableció en 0,05 y con dos colas. Todos los pacientes fueron incluidos en el análisis univariado. Todos los datos fueron procesados con el programa estadístico IBM SPSS Statistic, versión 16.

Este estudio fue aprobado por el Comité de Ética Científico del Servicio de Salud Metropolitano Oriente.

\section{Resultados}

Del grupo total de 120 pacientes con diagnóstico de BQ no-FQ adultos, el promedio de edad fue de $55 \pm 17,3$ años, que incluye a pacientes desde los 16 a los 86 años. En un 80,8\% correspondían a pacientes de género femenino. Las características generales de los pacientes se muestran en la Tabla 1.

Se identificaron las siguientes etiologías:

a) Postinfecciosa: Secuelas de infecciones en la infancia.

b) Post-TBC: Secuelas de infección por M. tuberculosis. 
c) IDCV: Asociadas a inmunodeficiencia común variable.

d) Disquinesia ciliar: Incluye el síndrome de Kartagener.

e) Asociada a enfermedades del tejido conectivo: Artritis reumatoide y Síndrome de Sjögren.

f) Idiopática: Se desconoce la etiología. Las etiologías se resumen en la Tabla 2.

Tabla 1. Características de los 120 pacientes con bronquiectasias no fibrosis quística

\begin{tabular}{|lc|}
\hline Características & BQ no-FQ $(\mathbf{n}=\mathbf{1 2 0})$ \\
\hline Edad (años) & $55,2 \pm 17,4$ \\
Género femenino & $97(80,8 \%)$ \\
mMRC & $2,1 \pm 1,2$ \\
$\mathrm{VEF}_{1}$ & $60,9 \% \pm 29,2$ \\
FACED & $2,86 \pm 1,94$ \\
\hline
\end{tabular}

mMRC: Modified Medical Research Council (Escala de disnea); $\mathrm{VEF}_{1}$ : Volumen espiratorio forzado en el $1^{\text {er }}$ segundo. BQ: Broquiectasias; FQ: Fibrosis quística; \pm : Desviación estándar.
La etiología más frecuente es la postinfecciosa, principalmente secuelas de TBC e infecciones en la infancia, otras causas asociadas a IDCV, mesenquimopatía, disquinesia ciliar y Kartagener, con $30,8 \%$ de etiología no identificada.

De acuerdo al aislamiento de Pseudomonas aeruginosa en los cultivos de esputo, se dividieron dos grupos: aquellos "infectados crónicamente", es decir, que resultaron con al menos dos cultivos positivos para PA, (BQ con PA; $n=61$ ) y el grupo de los "no infectados" (BQ sin PA; $\mathrm{n}=59$ ) quienes no cumplían con la condición previamente mencionada. Se buscaron diferencias respecto a la disnea, función pulmonar, score de FACED e ingresos hospitalarios y los resultados se muestran en la Tabla 3.

No hubo diferencias entre los grupos en edad y género. $\mathrm{El} \mathrm{VEF}_{1}$ fue más bajo en el grupo con PA $(47,7 \pm 23,5$ versus $64,8 \pm 22,9)$. Se calculó el índice de riesgo FACED ( $\mathrm{VEF}_{1}$, edad, colonización, extensión lobar, disnea) con diferencias significativas en ambos grupos.

Tabla 2. Etiología de las bronquiectasias no fibrosis quística

\begin{tabular}{|lcc|}
\hline Etiología & BQ no-FQ $(\mathbf{n}=\mathbf{1 2 0})$ & $\mathbf{\%}$ \\
\hline Postinfecciosa & 37 & 30,8 \\
Post-TBC & 36 & 30,0 \\
Inmunodeficiencia común variable (IDCV) & 2 & 1,7 \\
Disquinesia ciliar & 5 & 4,2 \\
Asociada a enfermedad reumatológica & 3 & 2,5 \\
Idiopática & 37 & 30,8 \\
\hline
\end{tabular}

BQ: Bronquiectasias; FQ: Fibrosis quística; TBC: Tuberculosis.

Tabla 3. Diferencias entre los grupos con BQ no-FQ infectados crónicamente por PA versus BQ no-FQ no infectados por $P A$

\begin{tabular}{|lccc|}
\hline Variable & BQ con PA $(\mathbf{n}=\mathbf{6 1})$ & BQ $\sin$ PA (n= 59) & p \\
\hline Edad (años) & $56 \pm 16,7$ & $54,4 \pm 18,2$ & 0,62 \\
Género femenino & $48 / 61(78 \%)$ & $49 / 59(83 \%)$ & 0,64 \\
Disnea, mMRC & $1,68 \pm 1,1$ & $2,54 \pm 0,976$ & $<0,0001$ \\
VEF 1 (\% valor teórico) & $47,7 \pm 23,5$ & $64,8 \pm 22,9$ & $<0,0001$ \\
FACED (Score) & $4,2 \pm 1,5$ & $1,9 \pm 1,6$ & $<0,0001$ \\
Hospitalizaciones (n) & $2,0 \pm 4,2$ & $0,67 \pm 1,3$ & 0,018 \\
\hline
\end{tabular}

BQ: Bronquiectasias; FQ: Fibrosis quística; PA: Pseudomonas aeruginosa; mMRC: modified Medical Research Council; \pm : Desviación estándar. 


\section{Discusión}

Las bronquiectasias no-fibrosis quística en el adulto, se presentan en diversos grupos etarios, incluyendo jóvenes hasta adultos mayores, como se puede observar en nuestra serie de pacientes. Llama la atención el predominio de género femenino, tal como se ha observado en otras publicaciones ${ }^{2}$, sin existir aun una clara explicación al respecto.

Las diferentes etiologías encontradas en nuestro grupo difieren de otras series, principalmente en relación al origen postinfeccioso. En nuestros resultados separamos aquellos que correspondían a secuelas de TBC y secuelas de otras infecciones, pero si se muestran como sólo un grupo corresponden al $60,8 \%$, lo cual duplica a lo reportado por otros autores ${ }^{10,11}$. Sin embargo, estos estudios han sido realizados en una población diferente (población inglesa en los estudios citados). Por otro lado, según lo reportado por Athanazio, en un estudio multicéntrico de población latina, el subgrupo de pacientes de Chile, presenta una prevalencia similar ${ }^{7}$. Situación que nos debe hacer reflexionar respecto al control de las enfermedades infecciosas para evitar daños crónicos.

Otros autores han sugerido que dado diferencias en los criterios de inclusión, los pacientes catalogados de causa idiopática podrían corresponder a etiología postinfecciosa. Teniendo presente lo anterior, si sumamos el grupo de pacientes de causa postinfecciosa y aquellos considerados como de origen idiopático, corresponden a alrededor del $80 \%-90 \%$ en todas las series, lo que se repite en nuestro grupo ${ }^{11}$.

En un 30,8\% se desconoce la causa, situación similar evidenciada en otras publicaciones. Sin embargo, se debe realizar un estudio acucioso en búsqueda de etiologías potencialmente tratables o que tienen un manejo específico (como infección de micobacterias ambientales, inmunodeficiencias, enfermedades autoinmunes, etc.). Algunos estudios han comunicado que una búsqueda exhaustiva de la etiología, puede modificar el pronóstico y el manejo entre un 15 y $37 \%^{10,11}$.

Aunque escapa a los objetivos planteados en el presente estudio, surge la interrogante sobre el comportamiento de las BQ no-FQ en el contexto de secuelas de $\mathrm{TBC}$, que podría ser distinto a otras etiologías.

Respecto a la infección crónica por Pseudomonas aeruginosa, nuestros resultados confirman que los pacientes presentan peores resultados respecto a la sintomatología, función pulmonar y mayor puntaje en score de FACED, presentando mayor número de hospitalizaciones, aunque sin diferencias estadísticamente significativas respecto al grupo no infectado. Estos hallazgos revelan la importancia de solicitar cultivos de esputo a los pacientes con diagnóstico de bronquiectasias. Las recomendaciones actuales sugieren ofrecer la erradicación de Pseudomonas aeruginosa en pacientes con bronquiectasias ${ }^{2}$, aunque con baja evidencia, pero que se sustenta desde la fisiopatología de la enfermedad que se iniciaría por la alteración de la depuración o clearance mucociliar de las secreciones, que conducen a la colonización de la vía aérea, produciendo inflamación mediada principalmente por neutrófilos y posteriormente daño estructural del árbol bronquial, resultando en un círculo vicioso de infecciones recurrentes, pérdida de la función pulmonar y exacerbaciones ${ }^{12}$.

Con frecuencia se describe la colonización crónica por Haemophilus influenzae y Pseudomonas aeruginosa, y con menor frecuencia con Moraxella catarrhalis, Staphylococcus aureus y Enterobacterias $^{2}$. Sin embargo, la infección por PA es de particular relevancia, considerando que un metaanálisis que incluyó 3.683 pacientes reportó que se asocia a un riesgo aproximadamente tres veces mayor de muerte y un aumento en las admisiones hospitalarias y las exacerbaciones en BQ no-FQ en adultos 9 .

Aún no se ha demostrado que la erradicación de este microorganismo disminuya la gravedad de la enfermedad, y sería interesante conocer el comportamiento de estos pacientes, quedando como una inquietud para futuros estudios.

\section{Conclusiones}

Los pacientes con bronquiectasias con infección crónica por Pseudomonas aeruginosa tienen una enfermedad más severa, con $\mathrm{VEF}_{1}$ más bajo, mayor disnea, más hospitalizaciones y mayor índice de severidad de FACED, pero no está claro si la infección por Pseudomonas es un marcador de gravedad de la enfermedad o contribuye a la progresión de ella.

\section{Bibliografía}

1.- PASTEUR MC, BILTON D, HILL AT; on behalf of the British Thoracic Society Bronchiectasis (non-CF) Guideline Group. British Thoracic Society guideline for non-CFbronchiectasis. Thorax 2010; 65 Suppl 1: i1-58. doi: 10.1136/thx.2010.136119.

2.- POLVERINO E, GOEMINNE PC, MCDONNELL MJ, 
ALIBERTI S, MARSHALL SE, LOEBINGER MR, et al. European Respiratory Society guidelines for the management of adult bronchiectasis. Eur Respir J 2017; 50: 1700-629.

3.- VENDRELL M, DE GRACIA J, OLVEIRA C, ÁNGEL MARTÍNEZ M, GIRÓN R, MÁIZ L, et al. Diagnóstico $\mathrm{y}$ tratamiento de las bronquiectasias. Arch Bronconeumol 2008; 44: 629-40.

4.- CHALMERS JD, CRICHTON M, GOEMINNE PC, LOEBINGER MR, HAWORTH C, ALMAGRO M, et al. The European Multicentre Bronchiectasis Audit and Research Collaboration (EMBARC): experiences from a successful ERS Clinical Research Collaboration. Breathe 2017; 13: 180-92.

5.- CERECEDA PJ, SAMSO ZC, SEGURA WA, SANHUEZA OP. Bronquiectasias en adultos: Características clínicas Experiencia de 5 años 1998-2003. Rev Chil Enferm Respir 2005; 21: 171-8.

6.- FERNÁNDEZ VP, JIMÉNEZ PP. Bronquiectasias: ¿No es más una enfermedad huérfana? Rev Chil Enferm Respir 2016; 32: 169-77.

7.- ATHANAZIO R, PEREIRA MC, GRAMBLICKA G, CAVALCANTI-LUNDGREN F, DE FIGUEIREDO MF, ARANCIBIA F, et al. Latin America validation of FACED score in patients with bronchiectasis: an analysis of six cohorts. BMC Pulm Med [Internet].
2017 [citado en 2017]; 17. Disponible en: http://bmcpulmmed.biomedcentral.com/articles/10.1186/s12890017-0417-3

8.- MARTÍNEZ-GARCÍA MA, DE GRACIA J, VENDRELL RELAT M, GIRÓN R-M, MAIZ CARRO L, DE LA ROSA CARRILLO D, et al. Multidimensional approach to non-cystic fibrosis bronchiectasis: the FACED score. Eur Respir J 2014; 43: 1357-67.

9.- FINCH S, MCDONNELL MJ, ABO-LEYAH H, ALIBERTI S, CHALMERS JD. A Comprehensive Analysis of the Impact of Pseudomonas aeruginosa Colonisation on Prognosis in Adult Bronchiectasis. Ann Am Thorac Soc [Internet]. 2015 [citado el 8 de octubre de 2017]; Disponible en: http://www.atsjournals.org/doi/10.1513/ AnnalsATS.201506-333OC.

10.- PASTEUR MC, HELLIWELL SM, HOUGHTON SJ, WEBB SC, FOWERAKER JE, COULDEN RA, et al. An Investigation into Causative Factors in Patients with Bronchiectasis. Am J Respir Crit Care Med 2000; 162: 1277-84.

11.- SHOEMARK A, OZEROVITCH L, WILSON R. Aetiology in adult patients with bronchiectasis. Respir Med 2007; 101: 1163-70.

12.- POPPELWELL L, CHALMERS JD. Defining severity in non-cystic fibrosis bronchiectasis. Expert Rev Respir Med 2014; 8249-62.
Correspondencia a:

Dra. Patricia Fernández V

General Salvo 357, Comuna de Providencia,

Santiago, Chile.

Email: pafeva99@hotmail.com 